TEMI

\title{
L'igiene dei prodotti alimentari nella disciplina europea
}

Maria Pia Ragionieri*

In materia di igiene dei prodotti alimentari la normativa della Comunità Europea è costituita da tre regolamenti, 852, 853 e 854 del 2004.

Essi rappresentano l'insieme di atti normativi, proposti dalla Commissione Europea, che dispongono sui requisiti igienici degli alimenti. Essi realizzano un approccio sistematico all'igiene nella produzione degli alimenti, quale profilo fondamentale per la loro sicurezza ${ }^{1}$ :

Il primo regolamento, l'852/2004, dispone sull'igiene degli alimenti, il secondo, 1'853/2004 dispone specificamente sull'igiene degli alimenti di origine animale, il terzo, l' 854 , sull'organizzazione dei controlli ufficiali sui prodotti di origine animale destinati al consumo umano. A questi si aggiunge il reg. 183/2005 sull'igiene dei mangimi.

Concentriamo l'attenzione sul reg. 852/2004 perché di portata generale e perché si applica ai prodotti alimentari di origine vegetale, dato lo specifico interesse dell'Egitto per l'esportazione in Europa di prodotti ortofrutticoli. Tale Regolamento abroga e sostituisce la direttiva n. 43 del 1993.

La direttiva per garantire la salubrità dei prodotti aveva previsto l'applicazione di norme di igiene in tutte le "fasi" di preparazione, trasformazione, lavorazione, confezionamento, deposito, trasporto, distribuzione, manipolazione e vendita o fornitura al consumatore finale; e aveva previsto la necessità di basare le norme di igiene sul ricorso all'analisi, controllo e

*Università della Tuscia, Viterbo.

Il contributo riproduce il testo della relazione tenuta alla "Food Safety Conference" svoltasi presso la Ain Shams University, Il Cairo - Egitto, il 17 giugno 2007.

${ }^{1}$ In materia di igiene alimentare v.: Germanò (2007, p. 71); Costato (2004, p. 735); Germanò, Rook Basile (2006, p. 361); Germanò, Rook Basile (2005, p. 271), Borghi (2004, p. 754); Losavio (2004, p. 679); Forti (2005, p. 325).

Agricoltura - Istituzioni-Mercati, n. 1/2008 
valutazione dei rischi, attraverso il sistema dell'Haccp (Hazard Analysis and Critical Control Points) $)^{2}$.

Il nuovo regolamento 852 contiene due importanti novità.

La prima riguarda la tipologia dell'atto normativo adottato ossia l'adozione di un "regolamento" al posto di una direttiva, cioè l'adozione di un atto normativo direttamente applicabile in tutti gli Stati Membri e tale dunque da garantire un'applicazione uniforme in tutto il territorió dell'Unione Europea. Agli Stati Membri spetta unicamente il compito di prevedere le sanzioni in caso di violazione delle norme comunitarie.

La seconda novità riguarda l'ampliamento del campo di applicazione della disciplina dell'igiene dei prodotti alimentari, includendo anche il cosiddetto "settore primario", mentre con la precedente direttiva le misure previste comprendevano solo le fasi successive all'applicazione primaria.

Per produzione primaria, secondo la definizione datane dall'art. 3 del reg. 178/2002 (sulla sicurezza alimentare) ${ }^{3}$ si intendono "le fasi della produzione, dell'allevamento, della coltivazione dei prodotti primari, compresi il raccolto, la mungitura e la produzione zootecnica precedente alla macellazione e comprese la caccia, la pesca e la raccolta di prodotti selvatici".

Le norme che si applicano alla produzione primaria sono contenute nell'allegato I, parte A, punto 1 del reg. 852 .

Nell'ambito della produzione primaria vanno ricomprese:

le operazioni ad essa associate, ossia: il trasporto, il magazzinaggio e la manipolazione di prodotti primari sul luogo di produzione, a condizione che ciò non alteri sostanzialmente la loro natura;

il trasporto di animali vivi, ove necessario per gli obiettivi del Regolamento;

- in caso di prodotti di origine vegetale, prodotti della pesca e della caccia: le operazioni di trasporto per la consegna di prodotti primari, la cui natura non sia stata ancora sostanzialmente modificata.

Dunque concentrando l'attenzione sui prodotti di origine vegetale, rientrano nella produzione primaria: la produzione e coltivazione di cereali, frutta, ortaggi, erbe e funghi; la raccolta di prodotti selvatici come funghi e bacche, il loro trasporto, magazzinaggio e manipolazione senza alterazione sostanziale della loro natura, nell'azienda agricola e loro successivo trasporto in uno stabilimento.

${ }^{2}$ Sul sistema Haccp cfr. Costato (2006, p. 427); Germanò, Rook Basile (2002, p. 143).

${ }^{3}$ Per un commento articolo per articolo al regolamento $178 / 2002$ si veda Idaic (2003), in particolare sulle definizioni contenute all'art. 3 del regolamento cfr. il commento di Germanò, Rook Basile (2003, p. 157). Sulla valutazione e gestione del rischio, Costato (2007, p. 47). Cfr., in genere sulla legislazione alimentare nella UE, Borghi (2005), Goldoni, Sirsi (2005).
Abbiamo sottolineato che nell'ambito della produzione primaria i prodotti primari possono essere trasportati, immagazzinati e manipolati a condizione che ciò non alteri sostanzialmente la loro natura (all. I, part. A, punto I.1.a). Sotto questo profilo le operazioni destinate a migliorarne la presentazione come la defoliazione degli ortaggi, la cernita della frutta, l'essiccamento di cereali sono considerate operazioni consuete che non richiedono l'osservanza di prescrizioni di sicurezza alimentare oltre quelle della produzione primaria.

Nella produzione primaria, ai sensi dell'all. I (in particolare il punto 5 per prodotti di origine vegetale), i principali rischi igienici attengono a contaminazioni derivanti da aria, suolo, acqua, mangimi, fertilizzanti, prodotti fitosanitari e pesticidi, carenze nella pulizia degli impianti o dei contenitori per il trasporto, da contatti con animali o insetti o per uso di acqua contaminata, da carenze di requisiti sanitari del personale o carenze nella gestione dei rifiuti dell'impresa, ecc.

Nell'applicazione delle regole igieniche sono utilizzati manuali di corretta pratica operativa. Si tratta di guide pratiche, strumenti di natura volontaria.

I manuali sono elaborati dall'industria alimentare di concerto con rappresentanti dei gruppi che possono essere coinvolti come l'associazione dei consumatori, tenendo conto delle relative linee guida del Codex Alimentarius o sotto l'egida di uno degli organismi nazionali di normalizzazione (in Italia l'Uni).

La valutazione dell'idoneità dei manuali spetta agli Stati Membri che li reputano conformi, li inviano alla Commissione Europea che li registra e li mette a disposizione degli altri Stati.

Tali manuali indicano: il campo di applicazione, le definizioni e le istruzioni; prendono in considerazione i locali (descrizione delle strutture, delle procedure di disinfestazione, manutenzione ecc.); le attrezzature e gli strumenti (procedure di pulizia e manutenzione); la produzione (tutto il ciclo produttivo e distributivo); il personale (igiene della persona e formazione); le linee guida per la stesura dei piani di autocontrollo (costituzione del gruppo, descrizioni delle fasi del processo, individuazione dei pericolo); analisi dei rischi ed individuazione dei punti critici, azioni correttive e procedure di verifica. Così, noi in Italia abbiamo il manuale per il settore ortofrutticolo-agrumicolo, per i centri di lavorazione e confezionamento per prodotti ortofrutticoli freschi, surgelati, ecc.

Il reg. 852 prevede che possono essere elaborati manuali anche a livello comunitario, sulla cui validità si pronuncia il Comitato permanente per la catena alimentare e salute degli animali, che è organo ausiliario dell'Efsa - 
Nell'allegato 1, parte B del reg. 852 sono fornite indicazioni sulle informazioni che i manuali devono contenere in merito ai pericoli che possono verificarsi nella produzione primaria e nelle azioni ad esse associate e sulle azioni di controllo dei pericoli (come il controllo della contaminazione da micotossine, il giusto utilizzo di acqua, ecc.).

Riguardo alle operazioni riconducibili o meno alla produzione primaria, la trasformazione dei prodotti nell'azienda agricola (come l'estrazione di succo dalla frutta) "non rientra" tra le attività di produzione primaria e ad essa si applicano pertanto le prescrizioni d'igiene dell'All. II del regolamento e, per quanto riguarda i prodotti alimentari di origine animale, anche le prescrizioni contenute nel reg. 853/2004.

Altre operazioni, seppure effettuate nell'azienda agricola, possono alterare i prodotti e/o implicare nuovi rischi per il prodotto alimentare, come la pelatura delle patate, l'affettatura delle carote, l'insacchettatura delle insalate e l'applicazione di gas conservanti. Tali operazioni non possono essere considerate operazioni consuete a livello di produzione primaria, secondo le indicazioni fornite dal Documento di orientamento sull'applicazione del reg. 852 della Direzione generale salute e tutela dei consumatori della Commissione Europea (21 dic. 2005).

Strettamente collegate al settore della produzione primaria, perché il prodotto non viene trasformato, ma da essa distinte, sono anche le operazioni di mondatura, taglio, pulizia e confezionamento in contenitori di plastica o sacchetti chiusi, di prodotti ortofrutticoli freschi e crudi destinati al consumatore finale, operazioni poste in essere dalle industrie alimentari, che rientrano fra le "imprese alimentari" ai sensi del reg. 852 e del reg. 178/2002. A tali operazioni si applicano le norme di igiene contenute nell'All. II al regolamento. In tali casi i contaminanti possono riferirsi ad agenti microbici, fungini, ecc.

Sia nella produzione primaria che nelle attività successive, per applicarsi la normativa comunitaria in tema di igiene è sufficiente svolgere una fase delle attività previste perché ricorra "l’impresa alimentare".

${ }^{4}$ Sull'Autorità europea per la sicurezza alimentare v. Babuscio (2005); Germanò (2007, p. 36). ${ }^{5}$ Sull'art. 58 del regolamento si rimanda al commento di Carmignani (2003, p. 474). 
c) adozione e misure di controllo e sorveglianza di tali punti critici;

d) riesame periodico delle analisi dei rischi alimentari.

Infine, dobbiamo sottolineare che il rigore delle norme sull'igiene che abbiamo illustrato è stemperato dalla presenza nel testo delle disposizioni di termini "ove necessario", "ove opportuno" o "sufficiente". Così, ai sensi dell'art. 4, gli operatori del settore alimentare, solo se necessario adottano misure igieniche specifiche come quelle relative al rispetto dei criteri microbiologici, alle campionature e analisi, al rispetto dei requisiti in materia di controllo delle temperature degli alimenti, ecc. (Borghi, 2005).

Ciò comporta che spetta all'operatore del settore alimentare stabilire se una prescrizione sia necessaria oppure adeguata o sufficiente per il perseguimento degli obiettivi del regolamento. E nel fare ciò deve tener conto della natura del prodotto alimentare e deve giustificare la scelta operata. Tale giustificazione deve risultare dalla documentazione che ogni operatore deve predisporre.

In questa valutazione giocano un ruolo fondamentale proprio i manuali di corretta prassi operativa per indicare all'operatore la procedura migliore ad evitare rischi a livello di produzione e il rispetto dei principi Haccp.

Le norme sull'igiene si applicano, come noto, anche ai prodotti dei Paesi Terzi che sono destinati al mercato europeo. Per essi è ammessa la possibilità che siano ottenuti secondo regole igieniche equivalenti (art. 10 reg. 852 che si richiama all'art. 11 del reg. 178/02; v. Valletta, 2003, p. 227). La lett. e) art. 2 del regolamento precisa che per "equivalente" come riferito a sistemi diversi debba intendersi "capace di conseguire gli stessi obiettivi posti dal Regolamento".

L'art. 10 del reg. 852 deve essere letto in correlazione con l'art. 11 del reg. 178/02 ai sensi del quale solo i prodotti conformi alle disposizioni comunitarie possono essere immessi sul mercato europeo. Qualora si dovessero applicare disposizioni differenti, queste dovrebbero comunque garantire caratteristiche "almeno equivalenti" a quelle della disciplina comunitaria, riconosciute tali dalla Comunità Europea, o essere frutto di un accordo specifico tra la Comunità e il Paese esportatore. In quest'ultima ipotesi non si potrebbe comunque configurare una effettiva possibilità di deroga agli standard comunitari.

La responsabilità degli operatori alimentari è disciplinata dall'art. 19 reg. 178/02 (su cui v. Bruno, 2003, p. 277).

Desideriamo concludere con una sintesi dei richiami normativi in materia di igiene, tenendo presente quanto espressamente contenuto nel Docu- mento-guida della DG Salute e tutela dei consumatori della Commissione del 5 gennaio 2006 "Sulle questioni fondamentali relative ai requisiti di importazione e alle nuove regole sull'igiene alimentare e sui controlli".

Con specifico riguardo ai prodotti di origine vegetali, i requisiti richiesti

in merito all'igiene, sono contenuti negli artt. 3-6 del reg. 852/04.

Gli operatori dei Paesi Terzi devono rispettare:

a) ai sensi dell'art. 3 un generale obbligo di garantire che tutte le fasi della produzione, trasformazione e utilizzazione degli alimenti sottoposti al loro controllo soddisfino i requisiti di igiene prescritti;

b) ai sensi dell'art. 4 , p. 1 , nella produzione primaria e operazioni connesse, il rispetto dei requisiti previsti nella parte a), All. I del regolamento;

c) ai sensi dell'art. 4, p. 2, i requisiti previsti per le fasi successive a quella primaria dall'All. II del regolamento;

d) ai sensi dell'art. 4, p. 3, per alcuni prodotti, i requisiti microbiologici (reg. 2073/2005);

e) ai sensi dell'art. 5, le procedure basate sui principi Haccp;

f) ai sensi dell'art. 6, l'obbligo di notifica degli stabilimenti posti sotto il loro controllo.

Oltre a quelli descritti, per i prodotti di origine vegetale devono essere osservati i requisiti fitosanitari.

Riguardo alle procedure di importazione, spetta all'importatore assicurare la conformità ai requisiti richiesti dalla normativa comunitaria o alle condizioni riconosciute equivalenti dalla Comunità.

Gli alimenti di origine vegetale possono essere sottoposti a controlli dall'autorità competente ai sensi dell'art. $15, \S 1$, reg. 882/04. Tali controlli devono essere effettuati in conformità alle leggi nazionali dei diversi Stati Membri. Ciò può avvenire al momento d'ingresso, in libera pratica (ossia al momento della libera circolazione), al momento dei permessi di importazione, al momento del mercato al minuto, ecc.

Il reg. 183/05 completa il sistema, prescrivendo per gli operatori della produzione primaria dei mangimi il rispetto delle norme dell'All. 1 del regolamento e a tutti gli altri operatori del settore il procedimento Haccp. 